\title{
Seasonal Variations in Lead Release in Potable Water
}

Sheldon Masters ${ }^{*}$, Gregory J. Welter ${ }^{2}$, and Marc Edwards ${ }^{1}$

AUTHOR ADDRESS. 'Via Department of Civil and Environmental Engineering, Virginia Tech,

Blacksburg, VA 24061; ${ }^{2} \mathrm{O}^{\prime}$ Brien and Gere Engineers, Inc, Bowie, MD

${ }^{*}$ CORRESPONDING AUTHOR. Via Department of Civil and Environmental Engineering, Virginia

Tech, Blacksburg, VA 24061. Email: smasters@vt.edu; Phone: (330) 347-7825; Fax: (540) 231-7916

KEYWORDS. Solubility, lead, copper, summer, winter.

\section{Summary of Figures and Tables}

Figure S1 Providence, RI pipe loop schematic

Figure S2 Photograph of control pipe rack in Providence, RI pipe loop study

Figure S3 Frequency distribution of normalized total lead data from the Washington Aqueduct pipe loop study.

Figure S4 Frequency distribution of normalized total lead data from the Washington Aqueduct pipe loop study.

Figure S5 Dissolution of cerussite, hydrocerussite, plumbonacrite, chloropyromorphite, lead orthophosphate and lead oxide.

Figure S6 Relationship between dissolved lead concentration and (a) temperature, (b) $\mathrm{pH}$, (c) total chlorine and (d) orthophosphate.

Table S1 Synthetic Providence water recipe

Table S2 Summary of Washington Aqueduct water quality

Table S3 Providence finished water quality

Table S4 Effect of temperature on average soluble lead concentration ( \pm standard error) for representative lead solids after 6 hours

Table S5 Summary of regression coefficient for $\log _{10}$ transformed total lead data from Washington Aqueduct

Table S6 Summary of regression coefficient for $\log _{10}$ transformed dissolved lead data from Washington Aqueduct

Table S7 Comparison of average lead and copper at each site during the summer and winter 


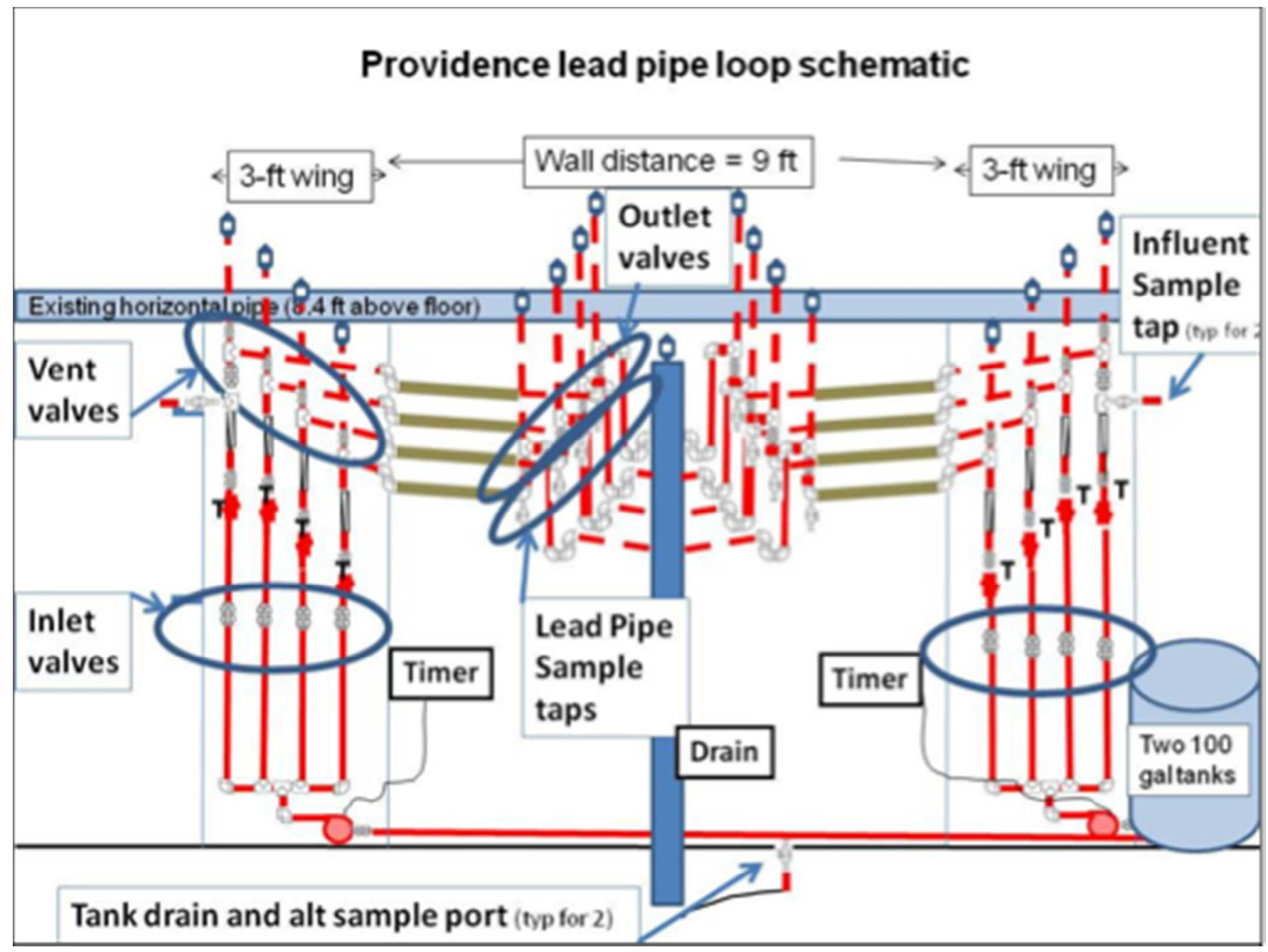

Figure S1. Providence, RI pipe loop schematic. Reproduced from reference 1. Copyright 2014 $O^{\prime}$ Brien and Gere. 


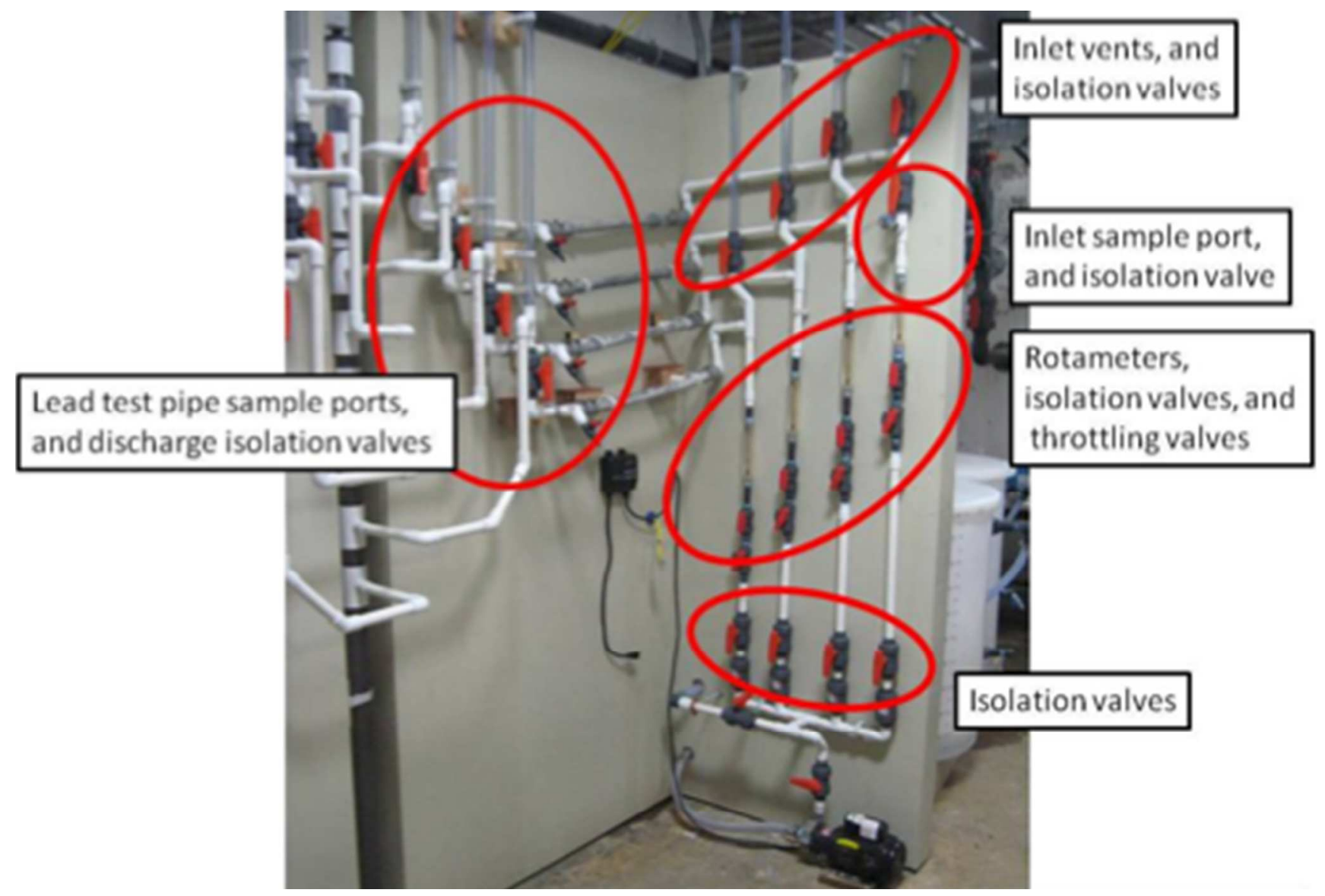

Figure S2. Photograph of control pipe rack in Providence, RI pipe loop study. Reproduced from reference 1. Copyright 2014 O’$^{\prime}$ Brien and Gere. 


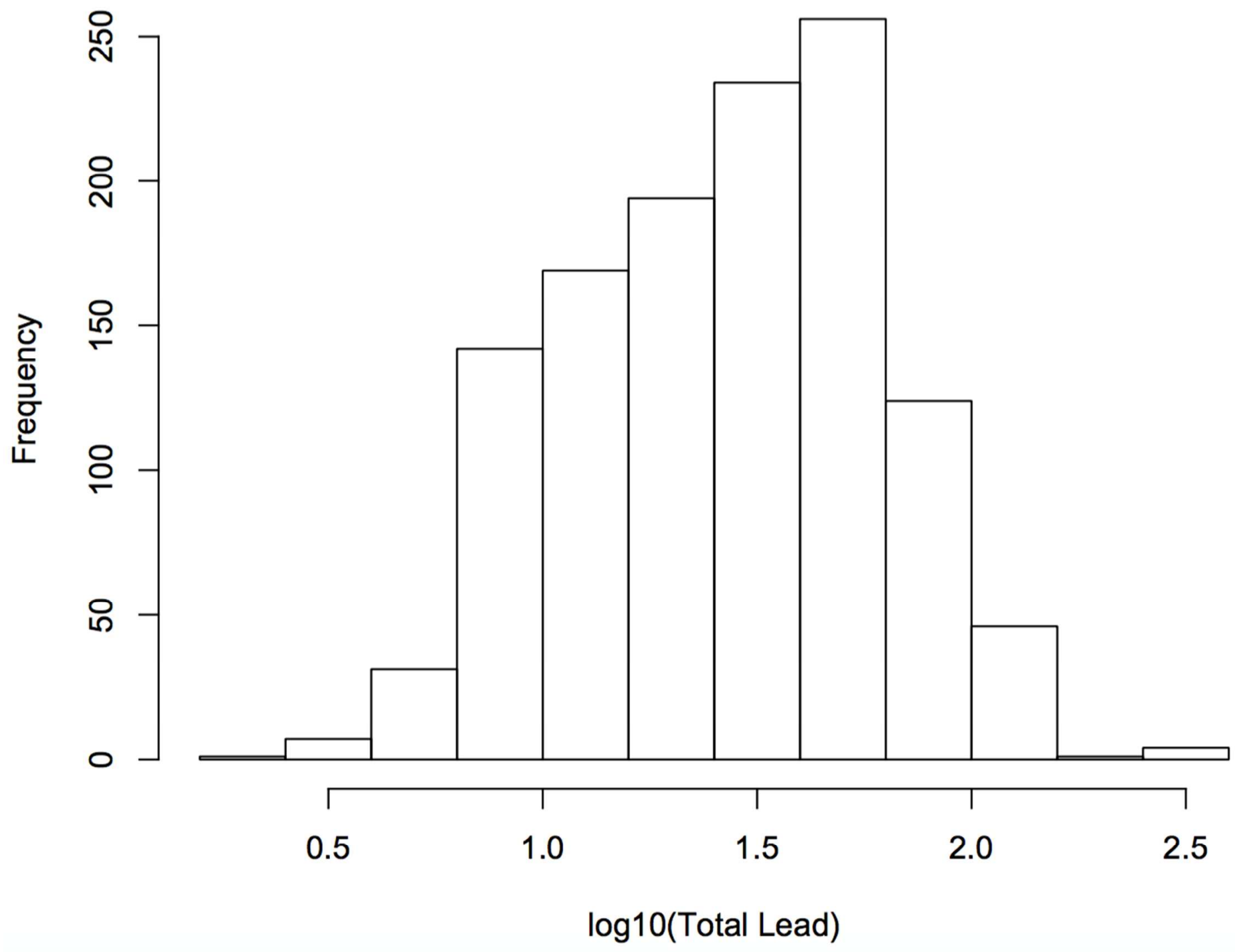

Figure S3. Frequency distribution of normalized total lead data from the Washington Aqueduct pipe loop study. 


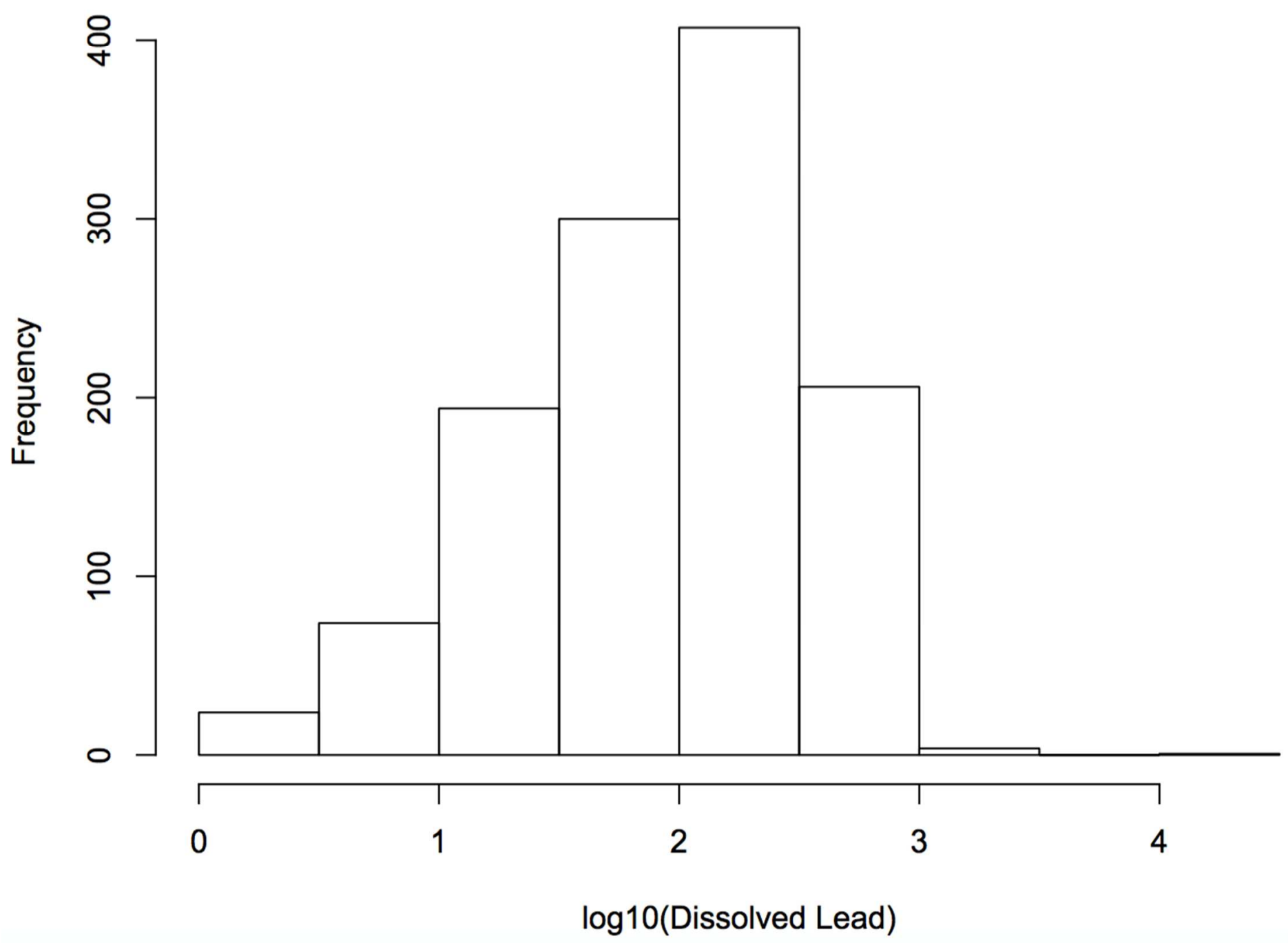

Figure S4. Frequency distribution of normalized total lead data from the Washington Aqueduct pipe loop study. 


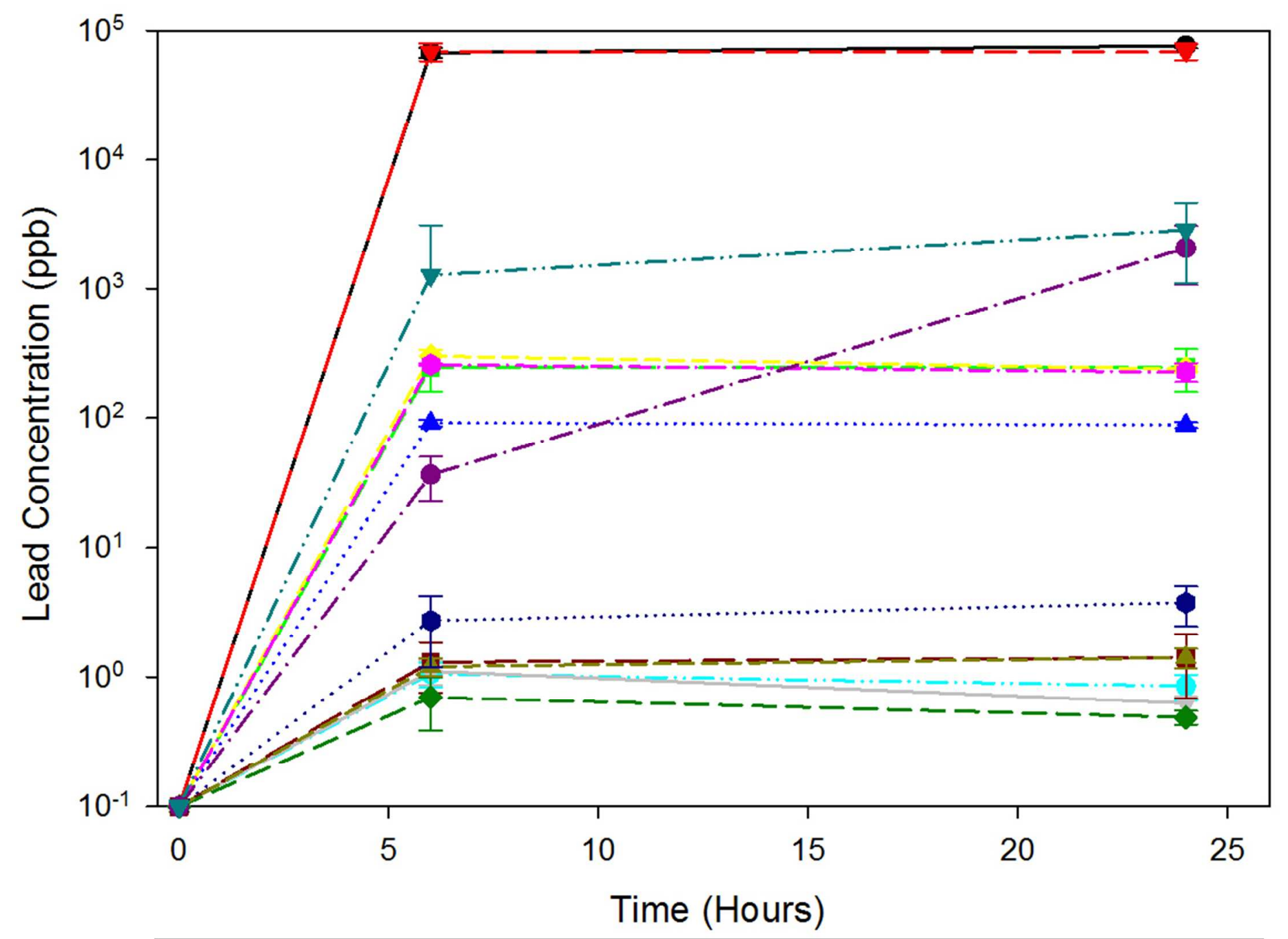

\begin{tabular}{|c|c|c|c|}
\hline 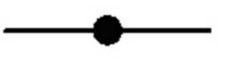 & ssite $4^{\circ} \mathrm{C}$ & - & orphite $20^{\circ} \mathrm{C}$ \\
\hline$-\rightarrow--$ & Cerussite $20^{\circ} \mathrm{C}$ & 1- - & Orthophosphate $4^{\circ} \mathrm{C}$ \\
\hline-- & Hydrocerussite $4^{\circ} \mathrm{C}$ & - - - & Orthophosphate $20^{\circ} \mathrm{C}$ \\
\hline & Hydrocerussite $20^{\circ} \mathrm{C}$ & $---1--$ & Lead Oxide $4^{\circ} \mathrm{C}$ \\
\hline$\cdots \wedge \cdots \cdots$ & Plumbonacrite $4^{\circ} \mathrm{C}$ & .... & Lead Oxide $20^{\circ} \mathrm{C}$ \\
\hline$-\cdot-\infty$ & Plumbonacrite $20^{\circ} \mathrm{C}$ & $-\cdot---$ & Lead Oxide $+\mathrm{NOM} 4^{\circ} \mathrm{C}$ \\
\hline$-\cdots-$ & Chloropyromorp & $-\cdots-\nabla \cdot \cdots$ & Lead Oxide + NOM $20^{\circ} \mathrm{C}$ \\
\hline
\end{tabular}

Figure S5. Dissolution of cerussite, hydrocerussite, plumbonacrite, chloropyromorphite, lead orthophosphate and lead oxide. Error bars represent standard error where $n$ is 3 . 

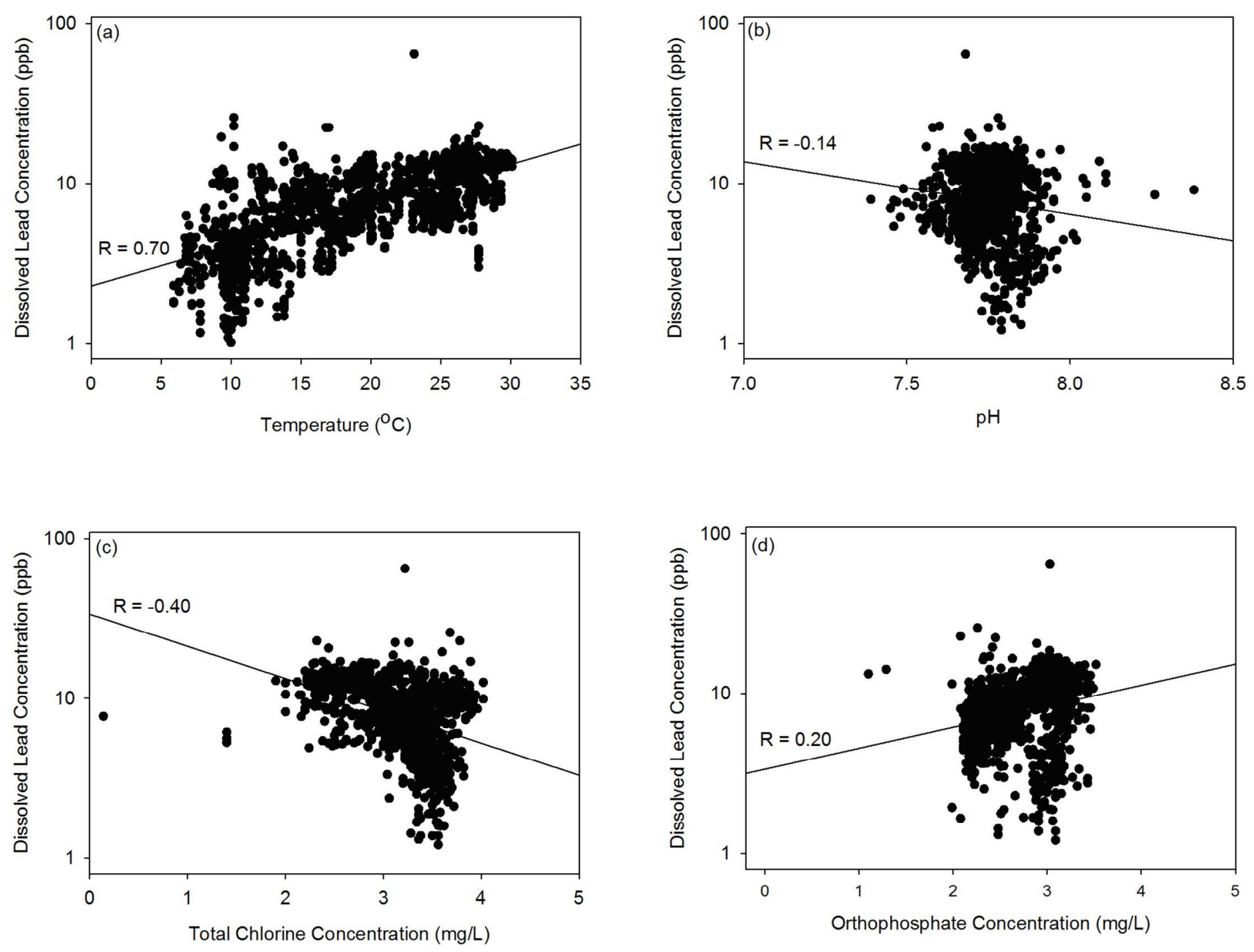

Figure S6. Relationship between dissolved lead concentration and (a) temperature, (b) $\mathrm{pH},(\mathrm{c})$ total chlorine and (d) orthophosphate. 
Tables

Table S1. Synthetic Providence water recipe

\begin{tabular}{|l|l|}
\hline \multicolumn{1}{|c|}{ Target water composition } & Conc. $(\mathrm{mg} / \mathrm{L})$ \\
\hline Total Alkalinity, as $\mathrm{CaCO}_{3}$ & 20 \\
\hline TDS & 113 \\
\hline Total Hardness, as $\mathrm{CaCO}_{3}$ & 38.5 \\
\hline Chloride & 19.4 \\
\hline Sulfate & 21.8 \\
\hline $\mathrm{pH}$ & 7.5 \\
\hline \multicolumn{1}{|c|}{ Chemicals added } & \\
\hline $\mathrm{NaHCO}_{3}$ & 11.76 \\
\hline $\mathrm{CaCl}_{2} \cdot 2 \mathrm{H}_{2} \mathrm{O}$ & 12.50 \\
\hline $\mathrm{MgSO}_{4} \cdot 7 \mathrm{H}_{2} \mathrm{O}$ & 55.92 \\
\hline $\mathrm{MgCl}_{2} \cdot 6 \mathrm{H}_{2} \mathrm{O}$ & 14.84 \\
\hline $\mathrm{NaCl}_{2}$ & 13.44 \\
\hline $\mathrm{NaNO}_{3}$ & 39.95 \\
\hline $\mathrm{Na}_{2} \mathrm{SiO}_{3}\left(\right.$ as $\left.\mathrm{SiO}_{2}\right)$ & 4.5 \\
\hline
\end{tabular}

Table S2. Summary of Washington Aqueduct water quality

\begin{tabular}{|l|l|}
\hline Parameter & Average \pm St. Dev. \\
\hline $\mathrm{pH}$ & $7.75 \pm 0.09$ \\
\hline Total Chlorine (mg/L) & $3.15 \pm 0.44$ \\
\hline Alkalinity as CaCO3 (mg/L) & $82.50 \pm 20.48$ \\
\hline $\begin{array}{l}\text { Dissolved Inorganic Carbon } \\
\text { (mg/L) }\end{array}$ & $21.43 \pm 4.89$ \\
\hline Calcium (mg/L) & $42.15 \pm 6.90$ \\
\hline Total Dissolved Solids (mg/L) & 193.84 .36 .19 \\
\hline Turbidity (NTU) & $3.47 \pm 4.64$ \\
\hline Ammonia (mg/L) & $0.92 \pm 0.10$ \\
\hline Nitrate (mg/L) & $1.67 \pm 0.56$ \\
\hline Phosphate (mg/L) & $2.75 \pm 0.41$ \\
\hline
\end{tabular}

Table S3. Providence finished water quality

\begin{tabular}{c|ccc}
\hline Parameter & Min & Max & Average \\
\hline $\mathrm{pH}$ & 10.2 & 10.5 & 10.4 \\
Alk. (mg/L as $\left.\mathrm{CaCO}_{3}\right)$ & 13.6 & 16.9 & 15.6 \\
Turbidity (NTU) & 0.10 & 0.13 & 0.12 \\
Total Hardness (mg/L) & 38.5 & 43.7 & 41.8 \\
\hline
\end{tabular}


Table S4. Effect of temperature on average soluble lead concentration ( \pm standard error) for representative lead solids after 6 hours

\begin{tabular}{|l|l|l|l|}
\hline Lead Solids & \multicolumn{2}{|c|}{ Concentration } & \\
\hline & $4{ }^{\circ} \mathrm{C}$ & $20^{\circ} \mathrm{C}$ & p-value \\
\hline Cerussite & $67793 \pm 5851$ & $68740 \pm 11052$ & 0.94 \\
\hline Hydrocerussite & $247 \pm 88$ & $303 \pm 36$ & 0.60 \\
\hline Plumbonacrite & $\mathbf{9 2} \pm 5.9$ & $\mathbf{2 6 0 \pm 4 . 9}$ & $<\mathbf{0 . 0 0 0 1}$ \\
\hline Chloropyromorphite & $1.1 \pm 0.2$ & $1.1 \pm 0.2$ & 0.85 \\
\hline Orthophosphate & $1.3 \pm 0.5$ & $0.7 \pm 0.3$ & 0.40 \\
\hline Lead Oxide & $1.2 \pm 0.2$ & $2.7 \pm 1.5$ & 0.43 \\
\hline Lead Oxide+NOM & $\mathbf{3 6 \pm 7}$ & $\mathbf{1 2 7 7 \pm 9 3 2}$ & $\mathbf{0 . 0 0 0 1}$ \\
\hline
\end{tabular}

Table S5. Summary of regression coefficient for $\log _{10}$ transformed particulate lead data from Washington Aqueduct

\begin{tabular}{|l|l|l|l|}
\hline Coefficients & $\begin{array}{l}\text { Coefficient } \\
\text { Estimate }\end{array}$ & $\begin{array}{l}\text { Standard } \\
\text { Error }\end{array}$ & p-value \\
\hline Intercept & -2.182 & 2.029 & 0.28 \\
\hline pH & 0.114 & 0.270 & 0.68 \\
\hline Temperature & $\mathbf{0 . 1 3 3}$ & $\mathbf{0 . 0 0 5}$ & $<\mathbf{0 . 0 0 0 1}$ \\
\hline Total Chlorine & $\mathbf{0 . 6 7 2}$ & $\mathbf{0 . 0 8 0}$ & $<\mathbf{0 . 0 0 0 1}$ \\
\hline Orthophosphate & -0.110 & 0.060 & 0.07 \\
\hline
\end{tabular}

Table S6. Summary of regression coefficient for $\log _{10}$ transformed dissolved lead data from Washington Aqueduct

\begin{tabular}{|l|l|l|l|}
\hline Coefficients & $\begin{array}{l}\text { Coefficient } \\
\text { Estimate }\end{array}$ & $\begin{array}{l}\text { Standard } \\
\text { Error }\end{array}$ & p-value \\
\hline Intercept & -0.694 & 1.15 & 0.55 \\
\hline $\mathrm{pH}$ & -0.001 & 0.153 & 0.993 \\
\hline Temperature & $\mathbf{0 . 0 6 9}$ & $\mathbf{0 . 0 0 3}$ & $<\mathbf{0 . 0 0 0 1}$ \\
\hline Total Chlorine & $\mathbf{0 . 3 3 8}$ & $\mathbf{0 . 0 4 5}$ & $<\mathbf{0 . 0 0 0 1}$ \\
\hline Orthophosphate & 0.144 & 0.034 & $<\mathbf{0 . 0 0 1}$ \\
\hline
\end{tabular}


Table S7. Comparison of average first draw lead and copper at each site during the summer and winter ${ }^{\Psi \Phi}$ (bold and italic indicates a statistically significant difference at the $95 \%$ confidence level)

\begin{tabular}{|c|c|c|c|c|c|}
\hline Sample Site & $\begin{array}{l}\text { First-draw } \\
\text { winter } \\
\text { average } \\
(\mathrm{ppb}) \\
\end{array}$ & $\begin{array}{l}\text { First-draw } \\
\text { summer } \\
\text { average } \\
(\mathrm{ppb})\end{array}$ & $\begin{array}{l}\text { First- } \\
\text { draw p- } \\
\text { value }\end{array}$ & $\begin{array}{l}\text { First-draw } \\
\text { winter } \\
\text { Temperature } \\
\text { Range }\left({ }^{\circ} \mathrm{C}\right) \\
\end{array}$ & $\begin{array}{l}\text { First-draw } \\
\text { summer } \\
\text { Temperature } \\
\text { Range }\left({ }^{\circ} \mathrm{C}\right) \\
\end{array}$ \\
\hline \multicolumn{6}{|c|}{ Dissolved Lead } \\
\hline Site 1 & 5.6 & 15.6 & 0.10 & $3.4-13.9$ & $16.5-20.3$ \\
\hline Site 2 & 5.4 & 2.1 & 0.47 & $15.3-18$ & $18.8-20.5$ \\
\hline Site 3 & 5.5 & 1.0 & 0.36 & $13.9-18.9$ & $18.7-23.2$ \\
\hline Site 4 & 2.8 & 1.9 & 0.64 & $10.0-13.6$ & $19.0-24.3$ \\
\hline Site 5 & 3.6 & 3.6 & 0.37 & $8.9-13.7$ & $23.6-24.4$ \\
\hline Site 6 & 9.2 & 4.9 & 0.70 & $12.8-23.5$ & $20.0-23.1$ \\
\hline Site 7 & 3.3 & 3.4 & 0.76 & $9.7-15.3$ & $19.5-22.7$ \\
\hline Site 8 & 2.5 & 4.1 & 0.75 & $10.7-16.0$ & $18.6-22.9$ \\
\hline \multicolumn{6}{|c|}{ Total Lead } \\
\hline Site 1 & 13.3 & 23.6 & 0.28 & $3.4-13.9$ & $16.5-20.3$ \\
\hline Site 2 & 52.3 & 12.1 & 0.37 & $15.3-18$ & $18.8-20.5$ \\
\hline Site 3 & 171.75 & 5.8 & 0.03 & $13.9-18.9$ & $18.7-23.2$ \\
\hline Site 4 & 17.0 & 15.7 & 0.34 & $10.0-13.6$ & $19.0-24.3$ \\
\hline Site 5 & 9.5 & 8.2 & 0.43 & $8.9-13.7$ & $23.6-24.4$ \\
\hline Site 6 & 31.9 & 33 & 0.88 & $12.8-23.5$ & $20.0-23.1$ \\
\hline Site 7 & 23.0 & 7.6 & 0.06 & $9.7-15.3$ & $19.5-22.7$ \\
\hline Site 8 & 48.1 & 18.0 & 0.59 & $10.7-16.0$ & $18.6-22.9$ \\
\hline \multicolumn{6}{|c|}{ Dissolved Copper } \\
\hline Site 1 & 9.0 & 4.2 & 0.17 & $3.4-13.9$ & $16.5-20.3$ \\
\hline Site 2 & 28.1 & 26.6 & 0.74 & $15.3-18$ & $18.8-20.5$ \\
\hline Site 3 & 10.2 & 2.2 & 0.11 & $13.9-18.9$ & $18.7-23.2$ \\
\hline Site 4 & 6.9 & 3.4 & 0.03 & $10.0-13.6$ & $19.0-24.3$ \\
\hline Site 5 & 20.4 & 8.9 & 0.04 & $8.9-13.7$ & $23.6-24.4$ \\
\hline Site 6 & 7.8 & 2.9 & 0.02 & $12.8-23.5$ & $20.0-23.1$ \\
\hline Site 7 & 16.5 & 3.75 & 0.01 & $9.7-15.3$ & $19.5-22.7$ \\
\hline Site 8 & 18.5 & 10.7 & 0.16 & $10.7-16.0$ & $18.6-22.9$ \\
\hline \multicolumn{6}{|c|}{ Total Copper } \\
\hline Site 1 & 14.1 & 5.7 & 0.12 & $3.4-13.9$ & $16.5-20.3$ \\
\hline Site 2 & 445.3 & 374.2 & 0.94 & $\begin{array}{l}15.3-18 \\
\end{array}$ & $18.8-20.5$ \\
\hline Site 3 & 88.59 & 6.0 & 0.02 & 13.9-18.9 & $18.7-23.2$ \\
\hline Site 4 & 19.6 & 9.8 & 0.19 & $10.0-13.6$ & $19.0-24.3$ \\
\hline Site 5 & 37.5 & 15 & 0.04 & $8.9-13.7$ & $23.6-24.4$ \\
\hline Site 6 & 17.6 & 6.9 & 0.01 & $12.8-23.5$ & $20.0-23.1$ \\
\hline Site 7 & 34.6 & 5.2 & 0.01 & $9.7-15.3$ & $19.5-22.7$ \\
\hline Site 8 & 137.3 & 35.7 & 0.01 & $10.7-16.0$ & $18.6-22.9$ \\
\hline
\end{tabular}

\footnotetext{
$\Psi$ Winter is defined as December-March while summer is defined as June-September. $\Phi_{n}$ is between 2 and 11 .
} 
Table S8. Comparison of average service line lead and copper at each site during the summer and winter $^{\Psi \Phi}$ (bold and italic indicates a statistically significant difference at the $95 \%$ confidence level)

\begin{tabular}{|c|c|c|c|c|c|}
\hline Sample Site & $\begin{array}{l}\text { Service line } \\
\text { winter } \\
\text { average } \\
\text { (ppb) }\end{array}$ & $\begin{array}{l}\text { Service line } \\
\text { summer } \\
\text { average } \\
\text { (ppb) }\end{array}$ & $\begin{array}{l}\text { Service } \\
\text { line p- } \\
\text { value }\end{array}$ & $\begin{array}{l}\text { Service line } \\
\text { winter } \\
\text { Temperature } \\
\text { Range }\left({ }^{\circ} \mathrm{C}\right) \\
\end{array}$ & $\begin{array}{l}\text { Service line } \\
\text { summer } \\
\text { Temperature } \\
\text { Range }\left({ }^{\circ} \mathrm{C}\right) \\
\end{array}$ \\
\hline \multicolumn{6}{|c|}{ Dissolved Lead } \\
\hline Site 1 & 9.3 & 8.8 & 0.93 & $2.8-9.8$ & $15.1-19.5$ \\
\hline Site 2 & 12.3 & 35.7 & 0.35 & $7.8-14.7$ & $18.0-20.1$ \\
\hline Site 3 & 4.2 & 1.5 & 0.67 & $6.4-17.1$ & $17.9-22.4$ \\
\hline Site 4 & 7.3 & 21 & 0.02 & $7.7-10.8$ & $18.1-24.5$ \\
\hline Site 5 & 11.6 & 20.9 & 1.0 & $7.6-11.5$ & $22.2-25.2$ \\
\hline Site 6 & 19.8 & 59.3 & 0.01 & $9-13.4$ & $16.8-23.0$ \\
\hline Site 7 & 19.2 & 59.0 & 0.01 & $7.3-10.3$ & $16.8-20.6$ \\
\hline Site 8 & 12.6 & 47.3 & 0.01 & $11.1-12.8$ & $16.0-21.5$ \\
\hline \multicolumn{6}{|c|}{ Total Lead } \\
\hline Site 1 & 20.9 & 15.6 & 0.58 & $2.8-9.8$ & 15.1-19.5 \\
\hline Site 2 & 24.2 & 50.8 & 0.44 & $7.8-14.7$ & $18.0-20.1$ \\
\hline Site 3 & 16.7 & 11.67 & 0.10 & $6.4-17.1$ & $17.9-22.4$ \\
\hline Site 4 & 32.1 & 43 & 0.24 & $7.7-10.8$ & $18.1-24.5$ \\
\hline Site 5 & 26.5 & 35.4 & 1.00 & $7.6-11.5$ & $22.2-25.2$ \\
\hline Site 6 & 54.3 & 86 & 0.10 & $9-13.4$ & $16.8-23.0$ \\
\hline Site 7 & 38.1 & 74.3 & 0.01 & $7.3-10.3$ & $16.8-20.6$ \\
\hline Site 8 & 53.3 & 103.0 & 0.02 & $11.1-12.8$ & $16.0-21.5$ \\
\hline \multicolumn{6}{|c|}{ Dissolved Copper } \\
\hline Site 1 & 3.9 & \begin{tabular}{|l|l|}
1.3 \\
\end{tabular} & 0.03 & $2.8-9.8$ & $15.1-19.5$ \\
\hline Site 2 & 3.2 & 2.5 & 0.57 & $7.8-14.7$ & $18.0-20.1$ \\
\hline Site 3 & 1.7 & 1.1 & 0.26 & $6.4-17.1$ & $17.9-22.4$ \\
\hline Site 4 & 1.4 & 1.0 & 0.28 & $7.7-10.8$ & $18.1-24.5$ \\
\hline Site 5 & 2.7 & 5.6 & 0.62 & $7.6-11.5$ & $22.2-25.2$ \\
\hline Site 6 & 2.0 & 1.5 & 0.24 & 9-13.4 & $16.8-23.0$ \\
\hline Site 7 & 2.3 & 1.1 & 0.06 & $7.3-10.3$ & $16.8-20.6$ \\
\hline Site 8 & 3.6 & 1.8 & 0.03 & $11.1-12.8$ & $16.0-21.5$ \\
\hline \multicolumn{5}{|c|}{ Total Copper } & \\
\hline Site 1 & 5.2 & \begin{tabular}{|l|l|}
1.5 \\
\end{tabular} & 0.16 & $2.8-9.8$ & $15.1-19.5$ \\
\hline Site 2 & 4.9 & 4.3 & 0.81 & 7.8-14.7 & $18.0-20.1$ \\
\hline Site 3 & 4.1 & 1.6 & 0.01 & $6.4-17.1$ & $17.9-22.4$ \\
\hline Site 4 & 2.4 & 1.2 & 0.94 & $7.7-10.8$ & $18.1-24.5$ \\
\hline Site 5 & 3.1 & 8.2 & 0.62 & 7.6-11.5 & $22.2-25.2$ \\
\hline Site 6 & 3.1 & 1.7 & 0.21 & $9-13.4$ & $16.8-23.0$ \\
\hline Site 7 & 3.8 & 1.4 & 0.04 & $7.3-10.3$ & $16.8-20.6$ \\
\hline Site 8 & 6.9 & 2.7 & 0.02 & $11.1-12.8$ & $16.0-21.5$ \\
\hline
\end{tabular}

$\Psi$ Winter is defined as December-March while summer is defined as June-September. $\Phi$ is between 2 and 11. 


\section{References:}

1. O'Brien \& Gere. Water Treatment Transition Lead Service Line Monitoring; 2014. 